\title{
Função renal de pacientes em uso de Everolimo associado a diferentes imunossupressores em terapia após transplante hepático.
}

\author{
Marcelo Augusto Scheidemantel Nogara1; Beatriz Bandeira de \\ Andrade2; Clarissa Novello Batzner ${ }^{3}$; Geovana Penteado Sartori4; \\ Leonardo Bandeira de Andrade ${ }^{5}$; Lucas Selistre Lersch ${ }^{6}$
}

\section{Resumo}

O estudo observou as possíveis diferenças significativas na taxa de filtração glomerular (TGF) de pacientes que utilizam o medicamento Everolimo (pertencente à classe dos inibidores do Mtor) associado a inibidores da calcineurina (Ciclosporina ou Tacrolimus), comparados aos que utilizam Everolimo associado a agentes antimetabólicos (Mofetil Micofenolato) e aos que utilizam Everolimo associado a outro inibidor do Mtor: o Sirolimo. Foram utilizados dados de prontuários de 23 pacientes em acompanhamento no Hospital Santa Isabel (HSI) de Blumenau - Santa Catarina (SC), em terapia imunossupressora pós-transplante hepático. A coleta de dados foi realizada através de busca ativa dos prontuários entre janeiro e setembro de 2014 no HSI - Blumenau (SC). Os pacientes responderam a um termo de consentimento livre e esclarecido antes da inclusão no estudo. A análise dos resultados ocorreu de setembro à dezembro de 2014, utilizando-se o programa Microsoft Excel 2010. A taxa de filtração glomerular estimada foi calculada pela fórmula MDRD, através do programa QxMD Software. O grupo de pacientes que apresentou piores taxas de filtração glomerular (TGF) foi o que estava em uso associado de Everolimo e Sirolimo (média TGF: $24,74 \mathrm{~mL} / \mathrm{min} / 1,73 \mathrm{~m}^{2}$ ). Pacientes em uso associado de Everolimo, Tacrolimus e Micofenolato apresentaram uma TGF média de 62,89 $\mathrm{mL} / \mathrm{min} / 1,73 \mathrm{~m}^{2}$, e o grupo em uso de Everolimo e Micofenolato, TGF média de $65,44 \mathrm{~mL} / \mathrm{min} / 1,73 \mathrm{~m}^{2}$. As melhores taxas de filtração glomerular estimadas encontraram-se entre os pacientes em associação terapêutica de Everolimo e Tacrolimus, com média de $101,57 \mathrm{~mL} / \mathrm{min} / 1,73 \mathrm{~m}^{2}$. Dez pacientes estavam com o prontuário incompleto em relação aos dados necessários para cálculo da função renal, e foram, portanto, excluídos desta análise do estudo. Com o presente estudo foi possível observar que o uso de 
Everolimo associado ao Tacrolimus apresentou uma melhora importante na TFG em relação às demais combinações estudadas. Contudo, sabemos das limitações impostas ao presente estudo devido à reduzida amostra. Ainda são necessários outros estudos a fim de encontrar a melhor associação farmacológica para o seguimento de pacientes transplantados.

Palavras Chave: imunossupressores; transplante hepático; taxa de filtração glomerular

\footnotetext{
${ }^{1}$ Graduação em Medicina pela Universidade Federal do Paraná - UFPR (1989) e Mestrado em Gastroenterologia Clínica pela Universidade de São Paulo - UNIFESP (1997). Atualmente atua como médico gastroenterologista na cidade de Blumenau/SC e como docente para o Curso de Medicina da Universidade Regional de Blumenau - FURB. <marcelonogara@gmail.com>

2 Graduanda de Medicina pela Universidade Regional de Blumenau - FURB. <andrade.beatriz@hotmail.com>

3 Graduanda de Medicina pela Universidade Regional de Blumenau - FURB. <clarissa.nbatzner@gmail.com>

4 Graduanda de Medicina pela Universidade Regional de Blumenau - FURB. <geovanasartori_1@hotmail.com>

5 Graduando de Medicina pela Universidade Regional de Blumenau - FURB. <andrade.leonardo@hotmail.com>

6 Graduando de Medicina pela Universidade Regional de Blumenau - FURB. <lucasselistre@hotmail.com>
} 